\title{
Inhalt, Vol. 17, No. 5, 1994
}

\section{Contents}

Übersichtsarbeiten

Genetische Prädisposition für das Mammakarzinom: Von epidemiologischen Studien zur molekularen Genetik

Krainer, $\mathrm{M}$

PSA in der Früherkennung des Prostatakarzinoms

Wirth, M., Manseck, A

Diagnostik und Therapie der Non-Hodgkin-Lymphome

Hense, J., Engelhard, M, Brittinger, G.

Review Articles

Genetic Predisposition for Breast Cancer:

From Epidemiologic Studies to Molecular Genetics

470 Krainer, M 470

PSA for Early Detection of Prostatic Carcinoma

478 Wirth, M., Manseck, A 478

Principles of Diagnosis and Therapy of Non-Hodgkin's Lymphomas

486 Hense, J, Engelhard, M., Brittinger, G 486

Original Articles

Simultaneous 5-FIuorouracil, Cisplatinum and Radiation Therapy for Patients with Locally Advanced Squamous Cell Carcinoma of the Esophagus

Hieber, U., Heim, M. E., Trux, F. A., Westerhausen, M., Diezler, P.,

Originalarbeiten

Simultane Therapie mit 5-Fluorouracil, Cisplatin und Bestrahlung bei Patienten mit lokal fortgeschrittenem Plattenepithelkarzinom des Ösophagus

Hieber, U., Heim, M. E., Trux, E A., Westerhausen, M., Diezler, P.,

Queißer,W. 498 Queißer, W.

198

Intraperikardiale Applikation von Mitoxantron in der Behandlung maligner Perikardergüsse Lentzsch, S., Reichardt, P., Gürtler, R., Dörken, B

\section{1 .}

Intrapericardial Application of Mitoxantrone for Treatment of Malignant Pericardia! Effusion Lentzsch, S., Reichardt, P., Gürtler, R., Dörken, B 504

Fludarabin ist eine effektive Substanz in der Therapie des Immunozytoms

Fenchel, K., Wijermans, P., Mitrou, P. S., Hoelzer, D.,

Bergmann, L 
Fludarabine is an Effective Agent in Immunocytic Lymphoma

Fenchel, K., Wijermans, P., Mitrou, P. S., Hoelzer, D.,

508 Bergmann, L

508

ALL-trans-retinoic-acid (ATRA) in der Behandlung der myelodysplastischen Syndrome:

Ergebnisse bei 5 Patienten

Baldus, M., Walter, H, Möller, M., Schürfeld, C, Brass, H 515

ALL-Trans Retinoic Acid (ATRA) in the Treatment of Myelodysplastic Syndromes: Results in 5 Cases

Baldus, M., Walter, H, Möller, M., Schürfeld, C, Brass, H 515

Assoziation von HLA-Antigenen mit verschiedenen Stadien des Harnblasenkarzinoms

Klän, R., Meier, Th., Wegner, H. E. H, Bünte, S.,

Dieckmann, K.-P

HLA Association with Different Stages of Transitional-Cell Carcinoma of the Bladder

Klän, R., Meier, Th., Wegner, H. E. H, Bünte, S.,

523 Dieckmann, K.-P.523

Zur Quantifizierung adhärenter Zellen der Kolonkarzinom-Zellinie HT29 auf extrazellulären Matrixproteinen: Vergleich einer isotopischen, einer fluorometrischen und einer kolorimetrischen Methode

Koenigsmann, M., Streit, M., Kreuser, E.D., Harms, U., Schulz, J.,

Quantifying Cell Adhesion of the Colon Cancer Cell Line HT29 to Extracellular Matrix Proteins:

Comparative Evaluation of an Isotopic, a Fluorometric, and a Colorimetric Method

Koenigsmann, M., Streit, M., Kreuser, E.D., Harms, U., Schulz, J.,

Mansmann, U., Thiel,E., Berdel,W.E 528 Mansmann, U, Thiel, E., Berdel, W.E.

518

Fortsetzung aufSeite 468

Continued on page 468

Band 17, Heft 5, Oktober 1994

International Journal for Cancer Research and Treatment

Inhalt

Contents

Brief an die Herausgeber

Letter to the Editors

Kattermann, R., zu: Kattermann, R., concerning:

Poley S, et al: ß2-Mikroglobulin-Bestimmung im Serum Poley S, et al: Serum B2microglobulin for staging and

zur Stadieneinteilung und Verlaufsbeobachtung multipler monitoring of multiple myelomas and other non-Hodgkin

Myelome und weiterer Nicht-Hodgkin-Lymphome 538 lymphomas 538 
Kongreßberichte $\quad 540$ Congress Reports

540

Klinische Information

Zur Messung von Lebensqualität in der Onkologie

Muthny, F.A

Clinical Information

Assessment of Life Quality in Oncology

547 Muthny, F.A

547

Buchbesprechung 558 Book Review

558

Industrieforum 559 Industrial Forum

55 ;

Mitteilungen onkologischer Gesellschaften 561 Reports of Oncological Societies

561

Impressum 465 Imprint 465

Inhaltsverzeichnis 466 Contents 466

Hinweise für Autoren 565 Instructions to Authors 565

Bibliographischer Hinweis: Inhaltsverzeichnisse dieser Zeitschrift erscheinen regelmäßig in current contents ${ }^{\circledR}$ sowie in anderen bibliographischen Diensten. 